\title{
Neuroethics and Brain Privacy: Setting the Stage
}

\author{
Jesper Ryberg ${ }^{1}$ (D)
}

Published online: 20 February 2017

(C) Springer Science+Business Media Dordrecht 2017

Despite the fact that ethics as an overall field of research has over the last couple of decades experienced the emergence of a large number of more specialized subdisciplines, there is little doubt that neuroethics—understood roughly as the study of ethical issues arising from what we can do to the brain and what we know about itconstitutes one of the most significant 'stories of success'.

Two decades ago the term 'neuroethics' had not yet been introduced in its disciplinary sense. However, shortly after the millennium a number of theorists began directing attention to its significance. Discussions cautiously emerged attempting to identify some of the important neuroethical challenges, and trying to define the area as a discipline in its own right. Even though the field in this early era in terms of research (unsurprisingly) evolved only slowly, there was a growing recognition of its future potentials. Some theorists talked of an 'unexplored continent lying between the populated shores of ethics and of neuroscience' ${ }^{1}$ or about 'neuroethics for the new millennium' (Roskies 2007). In his seminal work, Neuroethics: Challenges for the 21st century (2007), Neil Levy boldly predicted that neuroethics would undergo an explosive growth similar to that which bioethics has been undergoing since the late 1960s. Though it is obviously difficult (and unnecessary) to make this kind of comparison with any high degree of precision, it seems fair to hold that many of the early optimistic prophecies of the potentials of this new field have come true.

From having constituted only incipient and rough ideas in the minds of a relatively small group of enthusiastic theorists, neuroethics has today become a well-established and comprehensive area of research. Monographs, anthologies,

\footnotetext{
${ }^{1}$ The words of Al Jonson quoted by Adina Roskies (Roskies 2007, p. 12).

Jesper Ryberg

ryberg@ ruc.dk

1 Department of Philosophy, Roskilde University, Post Box 260, 4000 Roskilde, Denmark
} 
handbooks and numerous articles have been published; academic journals have been devoted specifically to this field; courses are being held at universities all around the world; researchers are being hired in permanent positions covering this field; and a vast number of seminars and conferences are continually being held. ${ }^{2}$ The field also has its own international organization. How can one explain this extraordinary development? Though it is not always easy to explain precisely why a scientific field has evolved in a certain way, in the present case it is not difficult to identify some inter-related features that have contributed to the remarkable growth of neuroethics.

The first explanation obviously has to do with the significant developments in neuroscience. Advances in new neuroscientific research tools and technologies for investigating the human brain have led to increasing insights into brain architecture and brain processes. Various sorts of imaging technologies-such as functional magnetic resonance imaging (fMRI), positron-emission tomography (PET), and electroencephalography (EEG) — have been pivotal in generating new neuroscientific knowledge. Moreover, modern neuroscience has also opened up the possibility of modifying or manipulating the human brain in various ways. It has for a long time been possible to alter the brain through pharmacology. However, more recent technologies designed for this purpose include both non-invasive methods, such as transcranial magnetic stimulation (TMS), and invasive methods such as deep-brain stimulation (DBS) or brain surgery. The colossal expansion that modern neuroscience has undergone, and the accompanying technological developments naturally give rise to normative considerations of how the new insights and methods should be used.

A second explanation, beyond the fact that neuroscience as a scientific field has been through a significant development, concerns the scope of the ways in which neuroscientific knowledge and technology can be used. Much neuroscientific research has been conducted within a medical framework. Insight into the function and structure of the brain has provided knowledge of the functionalities of the human body. And the fact that it has become possible to look non-invasively into the heads of patients has, in various ways, revolutionized clinical practice. Brain imaging is today playing a vital role in diagnostics, in the planning for operations and in several other ways. Furthermore, much research is being conducted on how both invasive and non-invasive methods for influencing the brain can be used to prevent or alleviate various diseases. However, had the results of modern neuroscience only had implications within a health-care context, then it is less likely that neuroethics would have undergone the above outlined explosive growth. Perhaps discussion of the ethical issues associated with new neuroscientific knowledge and technology would have been categorized simply as a division of medical ethics. However, this categorization is clearly inappropriate when it becomes clear that knowledge of the brain has potentials for application that reach far beyond a health-care framework. Modern neuroscience may have implications for many areas of social life and societal institutions. An example, one which has attracted much attention, is the use of neuroscience in a criminal justice context.

\footnotetext{
${ }^{2}$ A recent bibliography of neuroethics can be found in Roskies (2016). Other useful overviews of various neuroethical issues are those of Illes and Sahakian (2011) and Clausen and Levy (2015).
} 
Both monitoring and modification of the brain may be of relevance in relation to what constitutes the two main tasks of the criminal court, namely, to establish criminal guilt and to mete out appropriate punishments. For instance, one is (at least potentially) confronted with the possibilities of using neurotechnology as a means of detecting lies or biases, as a method of enhancing the memory of eye-witnesses or as a way of rehabilitating dangerous criminals. ${ }^{3}$ In the same vein, a plethora of possibilities and accompanying controversial questions arise from the use of neurotechnology in other important societal contexts. There is no need to elaborate with further examples. The point here is simply that the increasing realization of the fact that neuroscience and technology may have consequences for many areas of human life has contributed to the urgency for establishing a field of research addressing the questions of what constitutes desirable ways of applying neuroscientific knowledge and technology, and what should be regarded as applications that call for caution.

A third element in the explanation of the expansion of neuroscience has to do with the fact that the establishment of a new research discipline requires both that there is a sufficiently large number of questions that need to be addressed, and also that these questions are of a sufficiently challenging nature. A new research discipline presupposes theoretical challenges both in breadth and depth. As we have seen, the various ways in which neuroscientific knowledge and technology can be applied prompts questions concerning the desirability of the possible applications. And, as indicated, these questions very often do not allow for easy answers. Some questions constitute genuinely new challenges to be dealt with by the standard methods available in ethics. However, modern neuroscience has also contributed to a revivification and rephrasing of several of the classical theoretical challenges in ethics. For instance, how will new insight into brain processes affect the question as to whether people are responsible for the way they behave? And will neurotechnology enable us to qualify judgements with regard to the degree of responsibility that should be attributed to the performance of a particular act? If modern techniques for brain intervention can affect desires, memory, mood, character traits and so on, what does this imply for the conception of being a person or our view of personal identity? When will brain intervention constitute a threat to the autonomy of persons, and are there cases where such interventions can properly be held to enhance personal autonomy? All of these questions are challenging and involve well-known, but also controversial, concepts and theories. The fact that discussions of responsibility, personal identity, autonomy and other like concepts constitute the standard repertoire in ethics meant that, as the theoretical challenges related to these concepts were being reshaped in the light of new neuroscientific insights and technological possibilities, there was an already existing comprehensive theoretical interest to be canalized into neuroethics - thereby contributing, as a further reason, to the establishment and development of the field as a new ethical discipline.

The topic dealt with in this volume is just one of the plethora of issues that has attracted attention with the rise of neuroethics. It constitutes a clear example of how

\footnotetext{
${ }^{3}$ For an overview of some of the ethical challenges that arise in the intersection of neuroscience and criminal justice, see for instance Ryberg (2014) or Ryberg and Petersen (2017).
} 
already existing theoretical problems are being reframed in the light of the development of neurotechnology. Philosophers and other theorists have long been considering (in particular, over the last three or four decades) the conceptual and normative significance of privacy. While much of the traditional discussion of privacy has focused on the borders where our behaviour and thoughts meet the outside world, the inner life of our minds has usually been regarded as a bastion of privacy not calling for particular attention (see Roskies 2016). Realization of the fact that this bastion may be less impregnable than has usually been assumed has prompted very different reactions.

Some theorists have emphatically underlined that modern neurotechnology already constitutes (or at least will in the near future) a severe threat to privacy. In this view, the fact that there exist correlations between mental states and localized activities in the brain and that these activities may be visible on brain scans, thereby giving access to parts of an individual's mental life, calls for serious concern. Other theorists have adopted a less anxious approach, by emphasizing that, in the same way as some theorists have called for 'neuromodesty' in the current discussion of neuroscience and responsibility, there is reason to reject any kind of panic reaction when it comes to the implications neuroscience will have in terms of privacy.

However, no matter whether theorists hold an alarmist or more cautious view on the magnitude and urgency of the present and future threat that neurotechnology constitutes to privacy, it is a generally shared view that the major advances in neuroscientific research tools for investigating the functioning brain and the increasing insights into the various processes that shape human cognition and emotion give rise to a number of questions concerning privacy calling for closer theoretical scrutiny. For instance: does it make sense to hold that neurotechnological monitoring of an individual's mental life violates a right to brain privacy and, if so, what does such a right amount to? Do traditional normative theories of privacy manage to deal with the challenge presented by brain monitoring? Who should have access to information concerning an individual's brain (e.g. how should we react to a request for such information by employers and insurance companies)? Are there circumstances (e.g. in a criminal case) under which it is permissible for the state to force someone to undergo a brain scan? How should one react if brain scans for diagnostic use incidentally reveal important information about the individual's preferences or character traits?

The purpose of this special issue on Neuroscience and Brain Privacy is to consider some of these ethical challenges. However, as indicated, such questions cannot all — not even tentatively-be addressed within the scope of a few articles. More narrowly, the present volume explores some of the initial conceptual and normative questions that arise from the notion of brain privacy. The discussion is centred on the questions: to what does this aspect of privacy amount? Why is it valuable? And how ought it to be protected? More precisely, the issues dealt with within the ensuing articles are the following.

In the opening article, Adam Moore directs attention to the importance which he thinks privacy should have in relation to access to (and information about) an individual's brain. He presents a so-called control account of privacy and outlines a number of studies on both human and non-human animals, indicating the 
importance of privacy in relation to individual well-being and flourishing. On the ground of this view, he suggests a set of guidelines for adjudicating what he regards as an advancing tension between neuroscientific developments and privacy.

The contribution by Mark Tunick also has its main focus on the right to keep our inner thoughts to ourselves. Drawing on the spectacular criminal case of Gilberto Valle-known as the 'cannibal cop who', without acting out his fantasies, dreamed up (and corresponded with the like-minded) scenarios involving the kidnapping, torturing, and cannibalizing of women-Tunick suggests that individuals can have a right to privacy of their inner thoughts if a legitimate privacy interest is at stake and if this interest is not clearly outweighed by more compelling interests. Two of the main points of the contribution are first to underline that there is such an interest, and second to underscore the fact that it is highly important to distinguish between what goes on in our minds and what we put into practice.

While both Moore and Tunick direct attention to the significance of a right to privacy, Ryberg in his contribution adopts a much more sceptical stand. Even though he fully acknowledges that individuals have a privacy interest, this fact is not sufficient to sustain a right to mental privacy. What he argues is that, for such a right to be plausible, it would have to be specified in a way that is not too inclusive, that is, one which does not imply that many ordinary and (presumably) morally unproblematic ways of acting are suddenly turned into rights violations. Ryberg's purpose, therefore, is to examine whether it is possible to identify a morally relevant difference between standard instances of mind-reading (captured by the common view that people possess a 'theory of mind') and neuroscientific mind-reading. Several candidates for such a difference are examined, but it is concluded that none are morally convincing.

The contribution by Räikkä concerns an aspect of the value of privacy that was also touched upon by Tunick. More precisely, he deals with the relation between privacy and self-presentation (or, as he calls it 'self-enactment'). An often presented contention is that privacy is valuable because it enables an individual to govern the impressions that she gives and that this is important for the way she interacts with other people. The fact that neurotechnology may provide access to parts of our inner life thereby prompts the question as to whether the value of brain privacy, and the protection this sort of privacy calls for, can be explained by reference to the significance of self-enactment. While Räikkä suggests that brain imaging can threaten our capacity for self-enactment, he also underlines that the link between self-enactment and privacy is weaker than is commonly assumed and, hence, that the value of brain privacy cannot be grounded on the significance of being able to enact oneself.

Starting out by distinguishing between various ideas of privacy, LippertRasmussen in his contribution focuses on the sense of privacy which be believes is most clearly at stake in a discussion of brain-reading, namely 'information control privacy'. In line with the thoughts of Ryberg, he suggests that brain-reading does not threaten mind privacy in any ways that are qualitatively different from other mind-reading techniques. Furthermore, he argues that even though control privacy is often thought to promote or respect values such as intimacy and authenticity, it is nevertheless the case that even in an extreme scenario in which brain-reading 
technologies render our brains transparent to anyone, this need not clash with the values in question. He finishes by considering the lessons that can be drawn from his 'neuroimaging-friendly' conclusion.

The articles, as has been mentioned, leave many questions concerning the relation between neuroscience and privacy open for further scrutiny. But it is the modest hope that they will contribute to a discussion which is important and which, no doubt, will attract continued academic attention for years to come.

\section{References}

Clausen, J., and N. Levy (eds.). 2015. Handbook of neuroethics. Dordrecht: Springer.

Illes, J., and B. Sahakian (eds.). 2011. The Oxford handbook of neuroethics. Oxford: Oxford University Press.

Levy, N. 2007. Neuroethics: Challenges for the 21st century. Cambridge: Cambridge University Press. Roskies, A. 2007. Neuroethics for the new milllennium. In Defining right and wrong in brain science, ed. W. Glannon. New York, NY: Dana Press.

Roskies, A. 2016. Neuroethics. In Stanford encyclopedia of philosophy, ed. E. N. Zalta (online).

Ryberg, J. 2014. Neuroscience and criminal justice: Introduction. The Journal of Ethics 18 (2): 77-80.

Ryberg, J., and T. S. Petersen. 2017. Neuroethics and criminal justice. In A companion to applied philosophy, ed. K. Lippert-Rasmussen, K. Brownlee, and D. Coady. Oxford: Wiley. 